\title{
Effects of Acute Fatigue of the Hip Flexor Muscles on Hamstring Muscle Extensibility
}

\author{
by \\ José M. Muyor ${ }^{1}$, Francisco M. Arrabal-Campos ${ }^{2}$
}

The purpose of the present study was to evaluate the influence of acute fatigue of the hip flexor muscles on scores attained in tests frequently used in literature to measure hamstring muscle extensibility, namely the passive straight leg raise (PSLR), active straight leg raise (ASLR), passive knee extension (PKE), active knee extension (AKE), sit-and-reach (SR) and toe-touch (TT) tests. A total of seventy-five healthy and recreationally active adults voluntarily participated in this study. To reach fatigue, the participants actively lifted their legs alternately as many times as possible. In the passive tests, the results were $7.10 \pm 5.21^{\circ}$ and $5.68 \pm 4.54^{\circ}$ higher $(p<0.01)$ for PSLR and PKE tests, respectively, after acute fatigue. However, in the ASLR test, the results were lower post-fatigue than pre-fatigue (mean difference $\left.=-5.30^{\circ} \pm 9.51^{\circ} ; p<0.01\right)$. The AKE, SR and TT tests did not show significant differences between pre- and post-fatigue $(p>0.05)$. Moderate $(r=0.40)$ to high $(r=0.97)$ correlation coefficients were found, which were statistically significant among all the measured flexibility tests both pre-and post-fatigue. In conclusion, the active implication of the hip flexor muscles until reaching fatigue had acute influences on the results of the PSLR, PKE and ASLR tests, but not on the results of the AKE, SR and TT tests. It is recommended to use the AKE test to assess hamstring muscle extensibility in situations where athletes show fatigue in their hip flexor muscles.

Key words: flexibility; stretching; physical exercise; angular test; linear test.

\section{Introduction}

Stretching before or after physical exercise has frequently been observed in clinical settings and different populations such as clinicians, patients or athletes, who use stretching to prevent injuries, decrease soreness and improve performance (Andersen, 2005). The development and evaluation of hamstring muscle extensibility are important components of sports training and clinical practice. The lack of extensibility in the hamstring muscle has been associated with a higher risk of muscle strain injury (Hartig and Henderson, 1999) and spinal alterations, such as changes in lumbopelvic rhythm (Esola et al., 1996), greater thoracic kyphosis and lumbar flexion (Muyor et al., 2013) and lower back pain (Radwan et al., 2015). For these reasons, several tests have been proposed to determine hamstring muscle extensibility.

Great efforts have consistently been undertaken by researchers to determine which test is the most valid and feasible for measuring hamstring muscle extensibility among different groups of athletes, children and adolescents (Muyor et al., 2014b), university students (LópezMiñarro and Rodríguez-García, 2010) or the general population (Hui and Yuen, 2000; Muyor et al., 2014a), and to determine the differences and correlations among the different hamstring

\footnotetext{
1 - Faculty of Education Sciences. Laboratory of Kinesiology, Biomechanics and Ergonomics (KIBIOMER Lab.). University of Almería. Almería (Spain).

2 - Higher Engineering School. University of Almería. Almería (Spain).
} 
muscle extensibility tests (Radwan et al., 2015).

Among the most standard field-based tests used to assess hamstring muscle extensibility are the sit-and reach (SR) and toe-touch (TT) tests. Both tests are simple and safe to use, and their individual scores can be compared with normative data for a specific population, an age group and sex. However, these tests are considered to be indirect tests for measuring hamstring muscle extensibility as the results are the consequence of several factors, such as anthropometric dimensions (Simoneau, 1998), test protocols (Hui and Yuen, 2000) and methodological variables (McHugh et al., 2012). In this sense, several studies have analyzed the validity of the SR and TT tests and shown low to moderate correlation values (between $r=0.44$ and $\mathrm{r}=0.76$ ) relative to the passive straight leg raise (PSLR) test (Davis et al., 2008). Mier and Shapiro (2013) concluded that the SR score was largely independent on hip joint flexibility and was affected by multisegmental spine and pelvic flexibility. Muyor et al. (2014a) found that a pelvic tilt and lumbar flexion showed a greater variance explained by the score achieved on the SR and TT tests $(\mathrm{R} 2>0.75)$ than hamstring muscle extensibility (R2 < 0.65). López-Miñarro and Rodríguez-García (2010) reported that the hamstring criterion-related validity of the SR and TT tests was influenced by hamstring muscle extensibility. Kawano et al. (2010) showed that the position of the ankle influenced the distance reached on the SR test. However, no previous studies have evaluated whether hip muscle fatigue influenced the scores on the SR and TT tests.

Currently, the PSLR (Hui and Yuen, 2000; López-Miñarro and Rodríguez-García, 2010; Muyor et al., 2014b; Rolls and George, 2004) and active straight leg raise (ASLR) (Sporis et al., 2011) tests are considered the gold standards for measuring hamstring muscle extensibility as they only involve hip joint motion, compared with the SR and TT tests, which involve whole-body motion. However, for the PSLR and ASLR tests, it is very difficult to avoid movements of the pelvis, particularly when these tests are performed by participants with low hamstring extensibility (Congdon et al., 2005).

Other studies have used passive knee extension (PKE) (Muyor et al., 2013; Reurink et al.,
2013; Rolls and George, 2004) and active knee extension (AKE) (Davis et al., 2008; Norris and Matthews, 2005; Reurink et al., 2013) as specific tests to evaluate hamstring muscle extensibility. Both tests are similar, but the principal difference is that with the PKE test, the tester extends the knee passively until tension is felt or until the participants feel unacceptable tension in the hamstring area (Rolls and George, 2004). Norris and Matthews (2005) reported that AKE was a reliable measurement of hamstring muscle length. Reurink et al. (2013) found that PKE and AKE could reliably be used to assess flexibility in injured hamstrings, despite pain and discomfort during testing.

Nowadays, in the literature, the PSLR, ASLR, PKE, AKE, SR and TT tests are commonly used to evaluate hamstring muscle extensibility. Moreover, hip flexor muscles are involved in a large number of sports activities and in the active tests there is a direct implication of these muscles that could modify the results if they are fatigued. We hypothesized that fatigue of the hip flexor muscles would influence the score attained for hamstring muscle extensibility, depending on the test used. Specifically, in those tests where the hip is actively flexed, hip muscle fatigue could negatively influence the score reached. The purpose of the present study was to evaluate the influence of acute fatigue of the hip flexor muscles on the scores attained in tests frequently used in literature to measure hamstring muscle extensibility, such as the PSLR, ASLR, PKE, AKE, SR and TT tests.

\section{Material and Methods}

\section{Participants}

Thirty-eight male $(24.11 \pm 5.50$ years old, $67.76 \pm 13.53 \mathrm{~kg}, 169.23 \pm 6.09 \mathrm{~cm})$ and thirty-seven female $(22.35 \pm 2.52$ years old, $65.23 \pm 12.45 \mathrm{~kg}$, $169.69 \pm 7.71 \mathrm{~cm}$ ) healthy and recreationally active adults participated voluntarily in this study. Participants were excluded if they suffered from pain, had a history of orthopedic problems or hamstring injuries, or had experienced structural spinal pathologies. All of the participants were instructed to avoid strenuous training and physical activity for $24 \mathrm{~h}$ before the study.

All participants provided written consent before enrolling in the study. The Institutional Ethics Committee at the University of Almería 
(Spain) approved the study, which was conducted in accordance with the Declaration of Helsinki.

\section{Procedures}

This study used a randomized and quasiexperimental design with repeated measures. The experimental design consisted of evaluations of hamstring muscle extensibility when these muscles were relaxed and rested. Then, exercises were immediately performed to reach fatigue of the hip flexor muscles. Muscle fatigue was defined as the inability to successfully repeat a specific task (Williams and Ratel, 2009). In the current study, hip muscle fatigue was considered as the inability to perform more than one active hip flexion, lying supine. Finally, the same tests as at the beginning were performed to evaluate the new degree of hamstring muscle extensibility.

Six tests were used to measure hamstring muscle extensibility: the passive straight leg raise (PSLR), active straight leg raise (ASLR), passive knee extension (PKE), active knee extension (AKE), sit-and-reach (SR) and toe-touch (TT) tests. Some of the tests (active tests such as ASLR and AKE) directly involved the hip muscles and others (passive tests) depended more on the tension applied by the examiner than on hip muscle activation (Rolls and George, 2004). The SR and TT tests are affected by multisegmental spine and pelvic mobility (Constance and Shapiro, 2013; Muyor et al., 2014a), but not by hip muscle involvement (Constance and Shapiro, 2013). Therefore, we could determine the acute effects of fatigue of the hip flexor muscles on the scores attained on the tests used more often in the scientific literature to evaluate hamstring muscle extensibility.

At the beginning of the study, hamstring muscle extensibility was measured in both legs using the PSLR, ASLR, PKE and AKE tests. Additionally, the distance attained on the SR and TT tests was measured. Within each participant, all of these tests were performed in the same order in both conditions pre- and post- fatigue. However, between participants, the tests were performed in randomized order. Immediately after the measurements in pre-fatigue, the participant laid in the supine position on the floor, with the hands under the gluteus muscles. The participant actively lifted the legs alternatively, to an angular value of 65 degrees of hip flexion, as many times as possible. The velocity in each hip flexion was controlled by a metronome (KORG, Model MA-1, Tokyo, Japan) to 60 beats per minute. When he or she reached fatigue in the hip flexor muscles, namely, when the participant showed the inability to successfully perform the task within the given rhythm, the fatigue protocol was stopped and all the hamstring tests were immediately performed again in the same order than in the pre-fatigue condition, within a $5 \mathrm{~min}$ time limit to maintain the fatigue effect. All tests were performed by the same tester.

No warm-up or stretching exercises were performed by the participants before the first test measurements. All participants were examined while wearing sports shorts and barefoot. All of the measurements were obtained at the same time of the day and under the same environmental conditions (room temperature of $24^{\circ} \mathrm{C}$ ).

\section{Passive Straight Leg Raise Test}

This test was conducted following the same protocol as described by Muyor et al. (2014a). For the PSLR test (left and right legs), the participant was placed in the supine position with the lower extremities in $0^{\circ}$ hip flexion. While the participant was in the supine position, a UniLevel inclinometer (ISOMED, Inc., Portland, OR, USA) was placed over the distal tibia to measure the inclination. A lumbar protection support (Lumbosant, Murcia, Spain) was used to maintain neutral lumbar lordosis during the test (Muyor et al., 2014a). Thereafter, the participant's leg was lifted passively by the tester into hip flexion. The knee remained straight during the leg raise, while the pelvis and the other leg were fixed by an assistant tester to avoid a posterior pelvic tilt, as modifications in the pelvic positions had been reported to be factors that could affect the PSLR score (Bohannon et al., 1985). The endpoint for the straight leg raise was determined by one or both of the two following criteria: a) the participant reported pain in the hamstring muscle; and/or b) there was a palpable onset of pelvic rotation. Moreover, the ankle of the tested leg was restrained in maximum plantar flexion to avoid adverse neutral tension (McHugh et al., 2012).

\section{Active Straight Leg Raise Test}

The ASLR test was conducted using the identical methodology as on the PSLR test. The unique difference was that the participant had to lift his or her legs actively into hip flexion. The endpoint for the active straight leg raise was 
determined by the same criteria as described above for the PSLR.

\section{Passive Knee Extension Test}

This test was conducted following the same protocol as described by Muyor et al. (2013). The PKE was measured with the participant supine on the examining table and the opposite hip fully extended, maintained by an assistant tester to avoid a posterior pelvic tilt (Bohannon et al., 1985). The hip was flexed $90^{\circ}$ (as measured by the angle subtended by a line from the greater trochanter to the center of the femoral condyle and the horizontal plane). In this position, a unilevel inclinometer was placed over the distal tibia, and the knee was passively extended by the tester until moderate resistance was felt, or the participant reported pain in the hamstring muscle. The criterion score was the maximum angle (degree) read from the inclinometer at the point of maximum knee extension. The ankle of the tested leg was restrained in plantar flexion to avoid adverse neutral tension (McHugh et al., 2012).

\section{Active Knee Extension Test}

The AKE test was conducted with identical methodology to the PKE test. The unique difference was that the participant had to actively lift his or her legs into a maximum knee extension. The endpoint for the active knee extension was determined by the same criteria as described above for the PKE.

\section{Sit-and-reach test}

The participant was required to sit on the floor with the knees straight, legs together and the soles of the feet positioned flat against a sit-andreach box (ACUFLEX I Flexibility Tester, height: $32 \mathrm{~cm}$; PSYMTEC, Madrid, Spain). A standard meter rule was placed on the SR box, with a $0 \mathrm{~cm}$ mark representing the point at which the participant's fingertips were in line with his or her toes. With palms down, the participant placed the dominant hand on top of the other and was asked to bend forward as far as possible sliding the hands along the box, keeping the knees extended (Muyor et al., 2014b).

\section{Toe-Touch test}

The participant was placed in a standing position on the SR box, with the knees extended and fixed by a tester, and the feet spread to the width of his or her hips. A standard meter rule was placed on the SR box with a $0 \mathrm{~cm}$ mark representing the point at which the participant's fingertips were aligned with top surface of the sitand-reach box. From this position, the participant was asked to bend forward as far as possible, sliding the hands along the box to reach the maximal distance (Muyor et al., 2014a).

\section{Statistical Analyses}

The Kolmogorov-Smirnov test was used to test the normality of data distribution, whereas the homogeneity of variance was assessed using the Levene's test. No violations of the assumptions of normality and homogeneity were revealed. Parametric analyses were performed. Descriptive statistics (mean $\pm \mathrm{SD}$ ) were calculated for all variables. The dependent $t$-test was conducted to determine whether a significant difference existed between pre- and post-fatigue in the hip flexor muscles. Within group (pre- and post-) effect sizes (Cohen's d) were calculated using a pooled standard deviation. An effect size greater than 0.8 was considered large, approximately 0.5 was moderate, and less than 0.2 was small (Cohen, 1988). The relationships among the extensibility tests were determined by Pearson's correlation $(r)$.

Before comparing the effects of fatigue on the muscles evaluated, a $2 \times 2$ (male, female $X$ pre, post) analysis of variance (ANOVA) with repeated measures on the second dimension was used to determine whether there were differing responses considering sex. Additionally, paired ttest analysis was performed to determine differences in degrees of hamstring extensibility (left vs. right legs). There were no significant differences found between sexes or between hamstring extensibility of the legs, so the male and female scores and hamstring extensibility of both legs were combined for all of the analyses.

An alpha level of $p<0.05$ was considered statistically significant. The analyses were performed using the IBM SPSS statistics 22.0 software package.

\section{Results}

The mean values $\pm S D$, mean differences, $p$ values and effect sizes of the PSLR, ASLR, PKE, AKE, PKE, AKE, SR and TT tests pre- and postfatigue of the hip flexor muscles are shown in Table 1.

Paired $t$-test analysis reported statistically significant differences among the PSLR, ASLR 
and PKE. On the passive tests (PSLR and PKE), the results were higher post-fatigue than prefatigue $(p<0.01)$ with a moderate effect size $(d=$ 0.60 and 0.61 , respectively). Pre-fatigue, both tests showed similar results (mean difference $=0.82^{\circ} \pm$ $1.37^{\circ}$ ), and post-fatigue, both tests also showed similar results (mean difference $=0.60^{\circ} \pm 2.54^{\circ}$ ). However, on the ASLR test, the results were lower post-fatigue than pre-fatigue $(p<0.01)$ with a moderate effect size $(d=0.42)$. The AKE, SR and TT tests did not show statistically significant differences between pre- and post-fatigue $(p>$ $0.05)$ with a low effect size $(d<0.1)$ (Table 1$)$.

Table 2 shows moderate $(r=0.40)$ to high $(r=$ 0.97) correlation coefficients, which were statistically significant among all the measured flexibility tests both pre- and post-fatigue. The greatest correlations were observed between the $\mathrm{SR}_{\text {(pre) }}$ and $\mathrm{TT}_{\text {(post) }}$ tests, between the $\mathrm{TT}_{\text {(pre) }}$ and $\mathrm{TT}_{\text {(post) }}$ tests, and between the $\mathrm{SR}_{\text {(post) }}$ and $\mathrm{TT}_{\text {(post) }}$ tests; in all of these tests, the correlation was $r=$ 0.97. On angular tests, such as PSLR, ASLR, PKE and AKE tests (pre- and post-fatigue), there were statistically significant correlation coefficients among these tests. The AKE test, which showed no changes in results between pre- and postfatigue, showed a strong correlation between both conditions $(r=0.84)$ (Table 2).

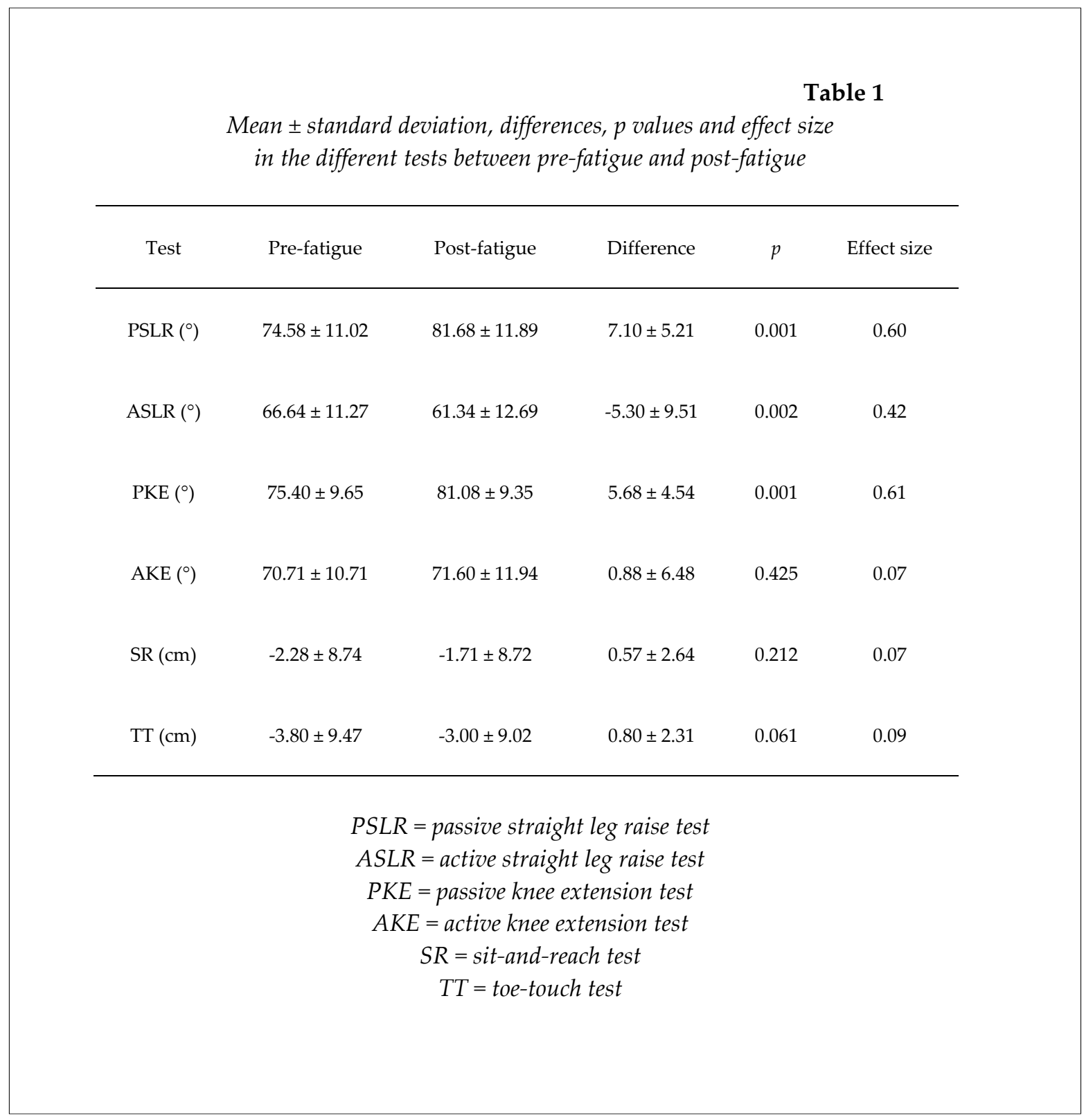




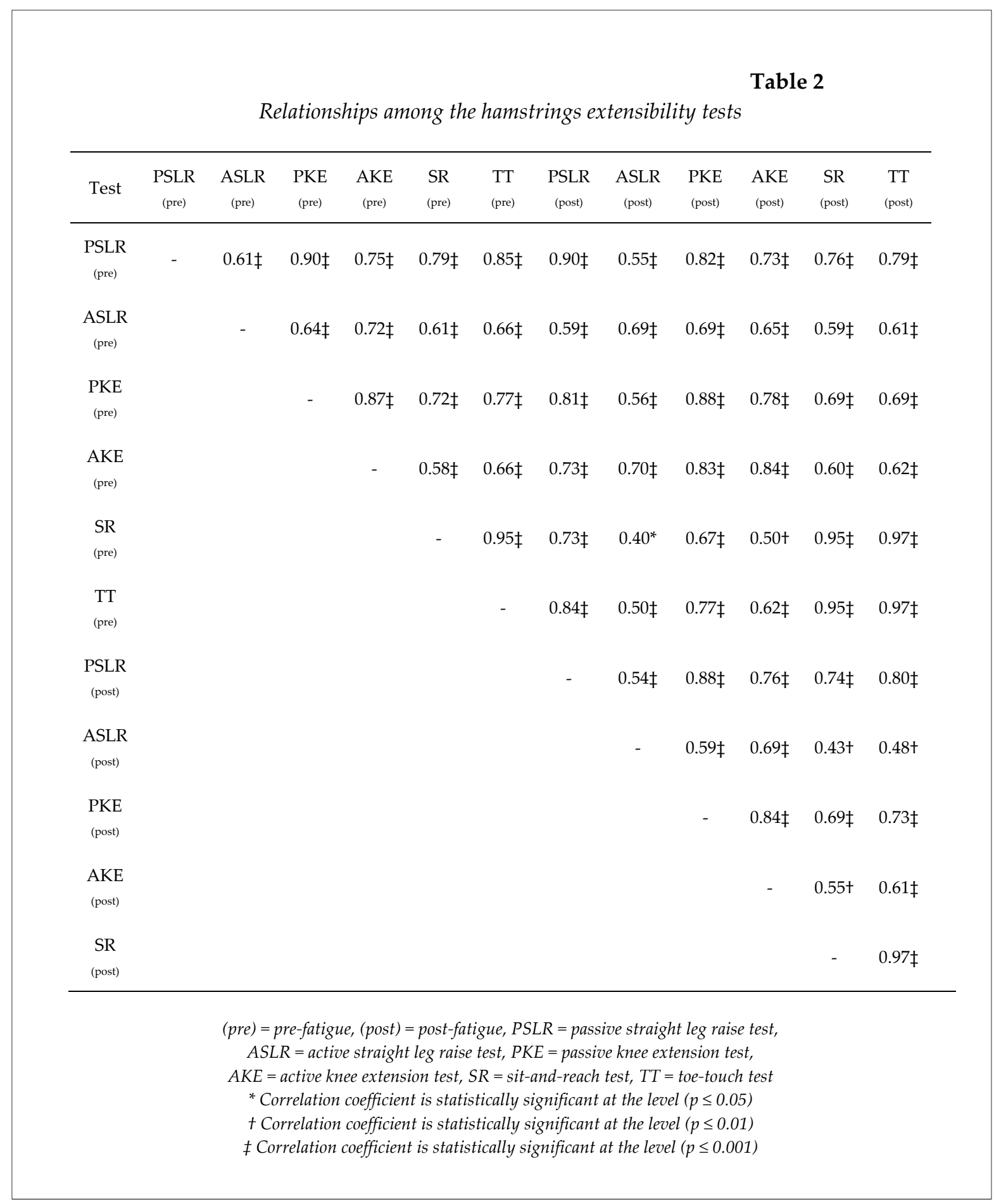

\section{Discussion}

Hamstring muscle extensibility is an important factor from the perspectives of health and sport performance in athletes. In scientific literature, many tests have been proposed to evaluate hamstring muscle extensibility ranging from linear tests, such as SR and TT tests, to angular tests, such as PSLR, ASLR, PKE and AKE tests. With both types of tests (linear and angular), there are some variables that can affect the results.

In the present study, the key finding was that on the passive hamstring muscle extensibility tests (PSLR and PKE), the scores were statistically higher post-fatigue than pre-fatigue (mean difference $=6.39^{\circ} \pm 4.87^{\circ} ; \mathrm{p}=0.000$ ). The greater hip ROM reached in the passive tests after fatigue condition could be due to changes in the mechanical properties (i.e. muscle stiffness or 
viscoelastic deformation) (Magnusson et al., 1996), changes in stretch reflex activity (Blazevich et al., 2014) or greater subjective tolerance to passive stretch (Magnusson et al., 2001). Stretch tolerance can be defined as the ability of a participant to tolerate an increase in the discomfort of the stretching procedure at the terminal range of motion (Reid and McNair, 2004). Reid and McNair (2004) reported that structural changes occurred in the stretched muscles after 6 weeks of a hamstring-stretching program. However, since our study did not include a stretching program, we agree with Magnusson et al. (1996) and Halbertsma and Göeken (1994) as, although they noted that passive resistive forces increased in range of motion after the training programs, they interpreted their finding as a change in stretch tolerance more than changes in the structural components of the muscle (Magnusson et al., 1996). Future studies are required to examine these potential mechanisms related to hamstring muscle extensibility after passive stretching repetitions.

However, in the current study, the ASLR test found lower degrees of hamstring muscle extensibility post-fatigue than pre-fatigue (mean difference $=5.30^{\circ} \pm 9.51^{\circ} ; p=0.002$ ). These results might be explained by fatigue in the hip flexor muscles as these muscles are highly activated $(\mathrm{Hu}$ et al., 2012), and this situation limited the active hip ROM.

In contrast with our results, Askling et al. (2010) found a greater (23\%) ROM with the ASLR test than with the PSLR test, perhaps because these authors evaluated the ASLR test at maximal velocity, and the inertial velocity might have facilitated attaining of a greater ROM. However, in the current study, the participants performed the ASLR test at a self-controlled velocity. In the same manner, Henderson et al. (2010) used active hip flexion range of motion (ASLR test) in a prospective study of a group of English Premier League soccer players. These authors found mean values between $64^{\circ}$ and $71^{\circ}$, similar to the angular values in our study.

Only the AKE, SR and TT tests showed similar values pre- and post-fatigue and without statistically significant differences $(p>0.05)$. With regard to the SR and TT tests, the results could be explained as these tests do not require active hip flexion, but the main movement is to perform trunk flexion. In fact, this is the main criticism of these tests when they are performed to evaluate hamstring muscle extensibility. Some studies have reported that the SR and TT tests could be used to evaluate a pelvic tilt and lumbar flexion capacity, but not hamstring muscle extensibility (Muyor et al., 2014a; Muyor et al., 2014b), yet with no previous fatigue in the hip flexor muscles. However, in the current study, there were moderate to high correlations between the SR and TT tests and angular tests, such as the PSLR, ASLR, PKE and AKE tests, pre- and post-fatigue.

Previous studies have used the AKE test as the gold standard for measuring hamstring muscle extensibility (Davis et al., 2008; Norris and Matthews, 2005; Reurink et al., 2013). In the present study, the AKE test was the only angular test that found similar results pre- and postfatigue.

Several limitations are present that could be addressed in future studies to complement the knowledge about this topic. First, to determine the specific level of uncomfortable sensation in the hamstring muscle during the passive tests, a visual analog pain scale could be used. Second, to determine the involvement of several muscles in the tests used, an electromyography system could be implemented. Third, evaluation of mechanical properties should be considered as it could result in greater hip ROM in the passive tests after acute fatigue in hip flexor muscles.

In conclusion, this study showed that active involvement of the hip flexor muscles until their fatigue had acute influence on the results of the PSLR, PKE and ASLR tests. In regard to the PSLR and PKE tests, the scores were higher, and on the ASLR test, the scores were lower postfatigue than pre-fatigue. However, there were no statistically significant differences on the AKE, SR and TT tests. Since angular tests are more adequate to evaluate hamstring muscle extensibility than linear tests, it is suggested to use the AKE test to evaluate hamstring muscle extensibility in situations where athletes show fatigue in their hip flexor muscles. 


\section{References}

Andersen JC. Stretching before and after exercise: Effect on muscle soreness and injury risk. J Athl Train, $2005 ; 3: 218-220$

Askling CM, Nilsson J, Thorstensson A. A new hamstring test to complement the common clinical examination before return to sport after injury. Knee Surg Sports Traumatol Arthrosc, 2010; 12: 1798-1803

Blazevich AJ, Cannavan D, Waugh CM, Miller SC, Thorlund JB, Aagaard P, Kay AD. Range of motion, neuromechanical, and architectural adaptations to plantar flexor stretch training in humans. J Appl Physiol, 2014; 5: 452-462

Bohannon R, Gajdosik R, LeVeau BF. Contribution of pelvic and lower limb motion to increases in the angle of passive straight leg raising. Phys Ther, 1985; 4: 474-476

Cohen J. Statistical power analysis for the behavioral science. Hillsdale, NJ: Lawrence Erlbaum Associates; 1988

Congdon R, Bohannon R, Tiberio D. Intrinsic and imposed hamstring length influence posterior pelvic rotation during hip flexion. Clin Biomech, 2005; 9: 947-951

Constance MM, Shapiro BS. Reliability of a computer software angle tool for measuring spine and pelvic flexibility during the sit-and-reach test. J Strength Cond Res, 2013; 2: 501-506

Davis DS, Quinn RQ, Whiteman CT, Williams JD, Young CR. Concurrent validity of four clinical tests used to measure hamstring flexibility. J Strength Cond Res, 2008; 2: 583-588

Esola MA, McClure PW, Fitzgerald GK, Siegler S. Analysis of lumbar spine and hip motion during forward bending in subjects with and without a history of low back pain. Spine, 1996; 1: 71-78

Halbertsma JP, Göeken LN. Stretching exercises: effect on passive extensibility and stiffness in short hamstrings of healthy subjects. Arch Phys Med Rehabil, 1994; 9: 976-981

Hartig DE, Henderson JM. Increasing hamstring flexibility decreases lower extremity overuse injuries in military basic trainees. Am J Sports Med, 1999; 2: 173-176

Henderson G, Barnes CA, Portas MD. Factors associated with increased propensity for hamstring injury in English Premier League soccer players. J Sci Med Sport, 2010; 4: 397-402

Hu H, Meijer OG, Hodges PW, Bruijn SM, Strijers RL, Nanayakkara PW, van Royen BJ, Wu W, Xia C, van Dieën JH. Understanding the Active Straight Leg Raise (ASLR): an electromyographic study in healthy subjects. Man Ther, 2012; 6: 531-537

Hui SS, Yuen PY. Validity of the modified back-saver sit-and-reach test: a comparison with other protocols. Med Sci Sports Exerc, 2000; 9: 1655-1659

Kawano MM, Ambar G, Oliveira BIR, Boer MC, Cardoso AP, Cardoso JR. Influence of the gastrocnemius muscle on the sit-and-reach test assessed by angular kinematic analysis. Rev Bras Fisioter, 2010; 1: 10-15

López-Miñarro PA, Rodríguez-García PL. Hamstring muscle extensibility influences the criterion-related validity of sit-and-reach and toe-touch tests. J Strength Cond Res, 2010; 4: 1013-1018

Magnusson SP, Julsgaard C, Aagaard P, Zacharie C, Ullman S, Kobayasi T, Kjaer M. Viscoelastic properties and flexibility of the human muscle-tendon unit in benign joint hypermobility syndrome. J Rheumatol, 2001; $12: 2720-2725$

Magnusson SP, Simonsen EB, Aagaard P, Sørensen H, Kjaer M. A mechanism for altered flexibility in human skeletal muscle. J Physiol, 1996; Pt 1: 291-298

McHugh MP, Johnson CD, Morrison RH. The role of neural tension in hamstring flexibility. Scand J Med Sci Sports, 2012; 2: 164-169

Mier CM, Shapiro BS. Sex differences in pelvic and hip flexibility in men and women matched for sit-andreach score. J Strength Cond Res, 2013; 4: 1031-1035

Muyor JM, López-Miñarro PA, Alacid F. The relationship between hamstring muscle extensibility and spinal 
postures varies with the degree of knee extension. J Appl Biomech, 2013; 6: 678-686

Muyor JM, Vaquero R, Alacid F, López-Miñarro PA. Criterion-related validity of sit-and-reach and toe-touch tests as a measure of hamstring extensibility in athletes. J Strength Cond Res, 2014a; 2: 546-555

Muyor JM, Zemková E, Stefánicová G, Kotyra M. Concurrent validity of clinical tests for measuring hamstring flexibility in school age children. Int J Sports Med, 2014b; 8: 664-669

Norris CM, Matthews M. Inter-tester reliability of a self-monitored active knee extension test. J Bodyw Mov Ther, 2005; 4: 256-259

Radwan A, Bigney KA, Buonomo HN, Jarmak MW, Moats SM, Ross JK, Tatarevic E, Tomko MA. Evaluation of intra-subject difference in hamstring flexibility in patients with low back pain: An exploratory study. J Back Musculoskelet Rehabil, 2015; 1: 61-66

Reid DA, McNair PJ. Passive force, angle, and stiffness changes after stretching of hamstring muscles. Med Sci Sports Exerc, 2004; 11: 1944-1948

Reurink G, Goudswaard GJ, Oomen HG, Moen MH, Tol JL, Verhaar JA, Weir A. Reliability of the active and passive knee extension test in acute hamstring injuries. Am J Sports Med, 2013; 41: 8

Rolls A, George K. The relationship between hamstring muscle injuries and hamstring muscle length in young elite footballers. Phys Ther Sport, 2004; 4: 179-187

Simoneau GG. The impact of various anthropometric and flexibility measurements on the sit-and-reach test. J Strength Cond Res, 1998; 4: 232-237

Sporis G, Vucetic V, Jovanovic M, Jukic I, Omrcen D. Reliability and factorial validity of flexibility tests for team sports. J Strength Cond Res, 2011; 4: 1168-1176

\section{Corresponding author:}

\section{José M. Muyor}

Edificio Humanidades A. Office 2.54. University of Almería. Ctra. Sacramento s/n. La Cañada de San Urbano. 04120. Almería (Spain).

Email: josemuyor@ual.es 